\title{
Biosynthesis of the 15-Membered Ring Depsipeptide Neoantimycin
}

\author{
Will Skyrud, ${ }^{\dagger, \bigcirc}$ Joyce Liu, ${ }^{\ddagger}, \bigcirc$ Divya Thankachan, ${ }^{\perp, \#}$ Maria Cabrera, ${ }^{\S}$ Ryan F. Seipke, ${ }^{*, \perp, \#}$ \\ and Wenjun Zhang ${ }^{*}, \|, \nabla_{\mathbb{C}}$
}

${ }_{\dagger}^{\dagger}$ Department of Chemistry, ${ }^{\ddagger}$ Department of Bioengineering, ${ }^{\S}$ Department of Molecular and Cell Biology, "Department of Chemical and Biomolecular Engineering, University of California, Berkeley, California 94720, United States

${ }^{\perp}$ Faculty of Biological Sciences, ${ }^{\#}$ Astbury Centre for Structural Molecular Biology, University of Leeds, Leeds LS2 9JT, United Kingdom

${ }^{\nabla}$ Chan Zuckerberg Biohub, San Francisco, California 94158, United States

\section{Supporting Information}

ABSTRACT: Antimycins are a family of natural products possessing outstanding biological activities and unique structures, which have intrigued chemists for over a half century. Of particular interest are the ring-expanded antimycins that show promising anticancer potential and whose biosynthesis remains uncharacterized. Specifically, neoantimycin and its analogs have been shown to be effective regulators of the oncogenic proteins GRP78/BiP and K-Ras. The neoantimycin structural skeleton is built on a 15 -membered tetralactone ring containing one methyl, one hydroxy, one benzyl, and three alkyl moieties, as well as an amide linkage to a conserved 3formamidosalicylic acid moiety. Although the biosynthetic gene cluster for neoantimycins was recently identified, the enzymatic logic that governs the synthesis of neoantimycins has not yet been revealed. In this work, the neoantimycin gene cluster is identified, and an updated sequence and annotation is provided delineating a nonribosomal peptide synthetase/polyketide synthase (NRPS/PKS) hybrid scaffold. Using cosmid expression and CRISPR/Cas-based genome editing, several heterologous expression strains for neoantimycin production are constructed in two separate Streptomyces species. A combination of in vivo and in vitro analysis is further used to completely characterize the biosynthesis of neoantimycins including the megasynthases and trans-acting domains. This work establishes a set of highly tractable hosts for producing and engineering neoantimycins and their C11 oxidized analogs, paving the way for neoantimycinbased drug discovery and development.

\begin{abstract}
A ntimycin (ant)-type depsipeptides are a large family of Inatural products widely produced by filamentous Actinobacteria. ${ }^{1,2}$ Antimycins are the archetypal members of this family and have been known for almost 70 years. ${ }^{3}$ They possess potent cytotoxicity because they bind to and inhibit mitochondrial cytochrome $\mathrm{c}$ reductase, the terminal step in respiration; ${ }^{4}$ this bioactivity is linked to a conserved 3formamidosalicylate moiety not observed elsewhere in nature. Antimycin is used commercially as a piscicide and is the active ingredient in Fintrol. Recently, antimycins were identified as inhibitors of the $\mathrm{Bcl}-2 / \mathrm{Bcl}-\mathrm{X}_{\mathrm{L}}$-related antiapoptotic proteins that are overproduced by cancer cells and confer resistance to chemotherapeutic agents whose mode of action is activation of apoptosis; it was also established that this antagonism is independent of its respiratory inhibitory activity. ${ }^{5}$

More than 40 naturally occurring derivatives of antimycin (nine-memberd ring) have been observed, and so-called "ringexpanded" members of the family have also been described. ${ }^{1}$ These include JBIR-06 (12-membered ring), ${ }^{6}$ neoantimycin (15-membered ring), ${ }^{7}$ and respirantin (18-membered ring). ${ }^{8}$
\end{abstract}

The hybrid nonribosomal peptide synthetase (NRPS)/ polyketide synthase (PKS) pathway dictating the biosynthesis of antimycins was identified recently, ${ }^{9}$ which facilitated the subsequent identification of biosynthetic gene clusters (BGCs) for ring-expanded members of the family. ${ }^{10,11}$ The biosynthesis of all ant-type depsipeptides is directed by a hybrid NRPS/PKS pathway that utilizes a 3-formamidosalicylate starter unit, followed by L-threonine, various $\alpha$-keto acids, and malonate derivatives to produce a linear depsipeptide, which is cyclized and released from the terminal module by a cis-acting thioesterase domain. The final cyclic depsipeptide is a macrocyclic ring varying in size depending on the number of NRPS modules, with each ring-expanded member of the family possessing one additional module. ${ }^{10}$

Neoantimycins (compounds 1-6; Figure 1) are of particular interest, because a C11 oxidized derivative of 3 named

Received: March 29, 2018

Accepted: April 25, 2018

Published: April 25, 2018 
A
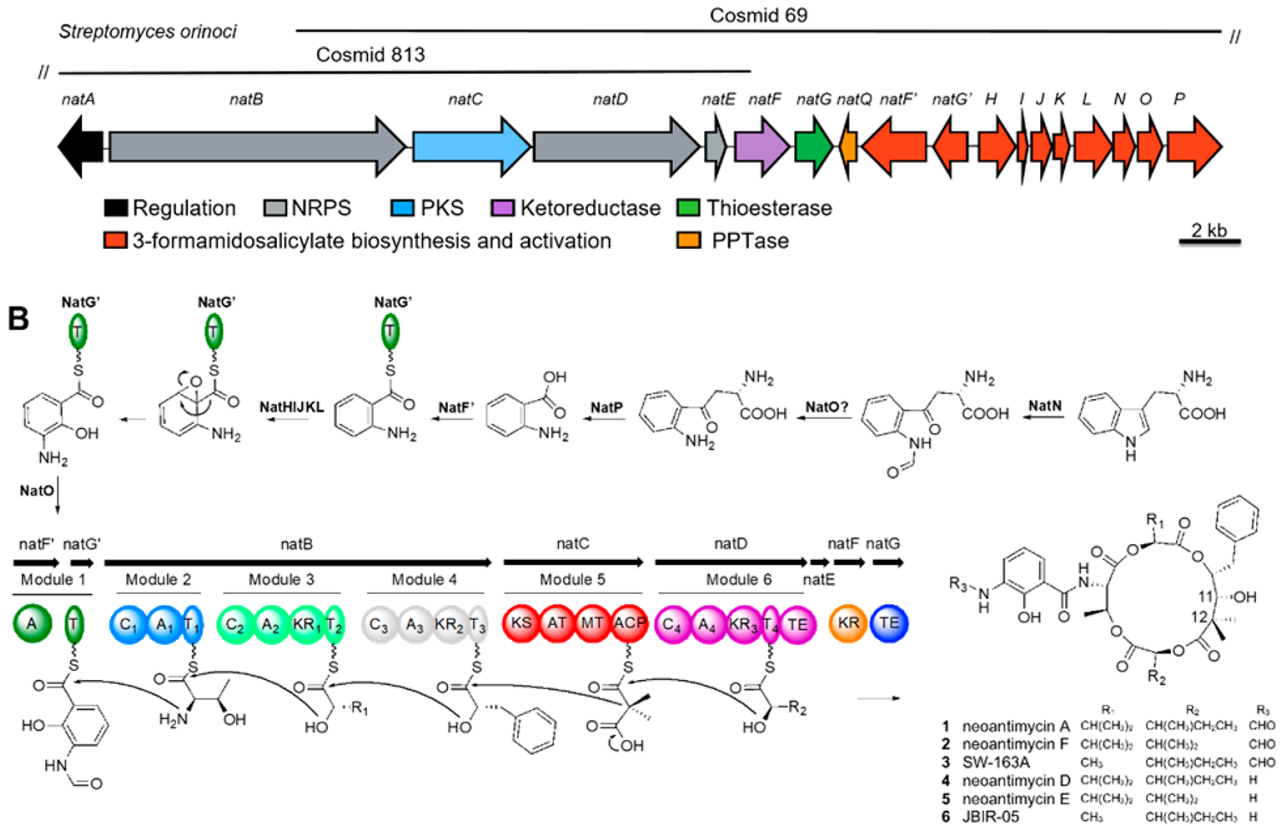

Figure 1. Biosynthesis of neoantimycins. (A) The neoantimycin biosynthetic gene cluster BGC in Streptomyces orinoci NRRL B-3379. The locations of Cosmid 69 and 813 are indicated by horizontal lines, and a double vertical hash indicates that the cosmid insert harbors additional DNA that falls outside the boundaries of this schematic. (B) The proposed biosynthetic pathway for neoantimycins. Structural variation arises from natural promiscuity of NRPS modules 3 and 6 as well as formylation of the starter unit. A, adenylation; T, thiolation; C, condensation; KR, ketoreductase; KS, ketosynthase; AT, acyltransferase; MT, methyltransferase; ACP, acyl-carrier protein; TE, thioesterase.

Table 1. Proposed Functions of Proteins Encoded by the Neoantimycin Biosynthetic Gene Cluster

\begin{tabular}{|c|c|c|c|c|c|}
\hline gene & $\begin{array}{c}\text { size, } \\
\text { aa }\end{array}$ & deduced role & protein homologue $^{a}$ & accession number & $\begin{array}{c}\text { identity/similarity } \\
\% / \%\end{array}$ \\
\hline NatA & 149 & $\begin{array}{l}\text { extracytoplasmic function RNA polymerase sigma } \\
\text { factor }\end{array}$ & AntA (S. blastmyceticus) & AGG37762.1 & $83 / 93$ \\
\hline NatB & 4542 & trimodular nonribosomal peptide synthetase & AntC (S. blastmyceticus) & AGG37764.1 & $68 / 76$ \\
\hline NatC & 1411 & unimodular polyketide synthase & hypothetical (S. albus sp. albus) & KUJ65684.1 & $75 / 82$ \\
\hline NatD & 2403 & unimodular nonribosomal peptide synthetase & $\begin{array}{l}\text { putative peptide synthetase ( } S . \\
\text { pyridomyceticus) }\end{array}$ & AEF33080.1 & $44 / 55$ \\
\hline NatE & 66 & MbtH-like protein & MbtH family protein (S. solWspMP-5a-2) & SCD38000.1 & $69 / 78$ \\
\hline NatF & 342 & ketoreductase & NAD-dependent epimerase (N. cosensis) & WP033408890.1 & $54 / 65$ \\
\hline NatG & 255 & thioesterase & hypothetical (S. caatingaensis) & WP049714988.1 & $69 / 78$ \\
\hline NatQ & 230 & phosphopantetheinyl transferase & EntD (Streptomyces sp. TLI 146) & PKV83804.1 & $59 / 66$ \\
\hline NatF $^{\prime}$ & 495 & Acyl-CoA ligase & CoA ligase (Streptomyces sp. ADM21) & AIF33754.1 & $81 / 88$ \\
\hline $\mathrm{NatG}^{\prime}$ & 79 & peptidyl carrier protein & AntG (S. blastmyceticus) & AGG37769.1 & $96 / 96$ \\
\hline NatH & 339 & multicomponent oxygenase & PaaG (S. albus sp. J1074) & AG192189.1 & $84 / 91$ \\
\hline Natl & 97 & multicomponent oxygenase & anti (S. blastmyceticus) & AGG37771.1 & $82 / 91$ \\
\hline NatT & 251 & multicomponent oxygenase & Paal (S. lincolnensis) & WP067444180.1 & $72 / 80$ \\
\hline NatK & 165 & multicomponent oxygenase & PaaJ (Streptomyces sp. ERV7) & OAR24946.1 & $81 / 89$ \\
\hline NatL & 366 & multicomponent oxygenase & AntL (S. blastmyceticus) & AGG37774.1 & $74 / 82$ \\
\hline NatN & 270 & tryptophan 2,3-dioxygenase & AntN (S. blastmyceticus) & AGG37776.1 & $79 / 87$ \\
\hline NatO & 274 & $N$-formylase & $\begin{array}{l}\text { alpha/beta hydrolase (streptomyces } s p \text {. } \\
\text { ERV7) }\end{array}$ & WP067162889.1 & $81 / 86$ \\
\hline NatP & 416 & kynureninase & AntP (S. blastmyceticus) & AGG37778.1 & $80 / 87$ \\
\hline
\end{tabular}

prunustatin A downregulates the expression of the major molecular chaperone GRP78/BiP, whose overproduction is required for cancer cell survival during hypoxic conditions caused by their rapid proliferation. ${ }^{12}$ Additionally, several neoantimycin derivatives displayed nanomolar potency in causing the mislocalization of oncogenic K-Ras, a GTPase that regulates cell growth and proliferation in numerous cancers. $^{13,14}$ Thus, neoantimycin is an exciting anticancer lead compound for the treatment of diverse cancers. Natural product lead compounds are difficult to derivatize synthetically, and a powerful alternative approach to this is bioengineering. We therefore sought to robustly characterize the biosynthesis of neoantimycin as the first step toward establishing a platform for biologically expanding the chemical space of this compound class. 

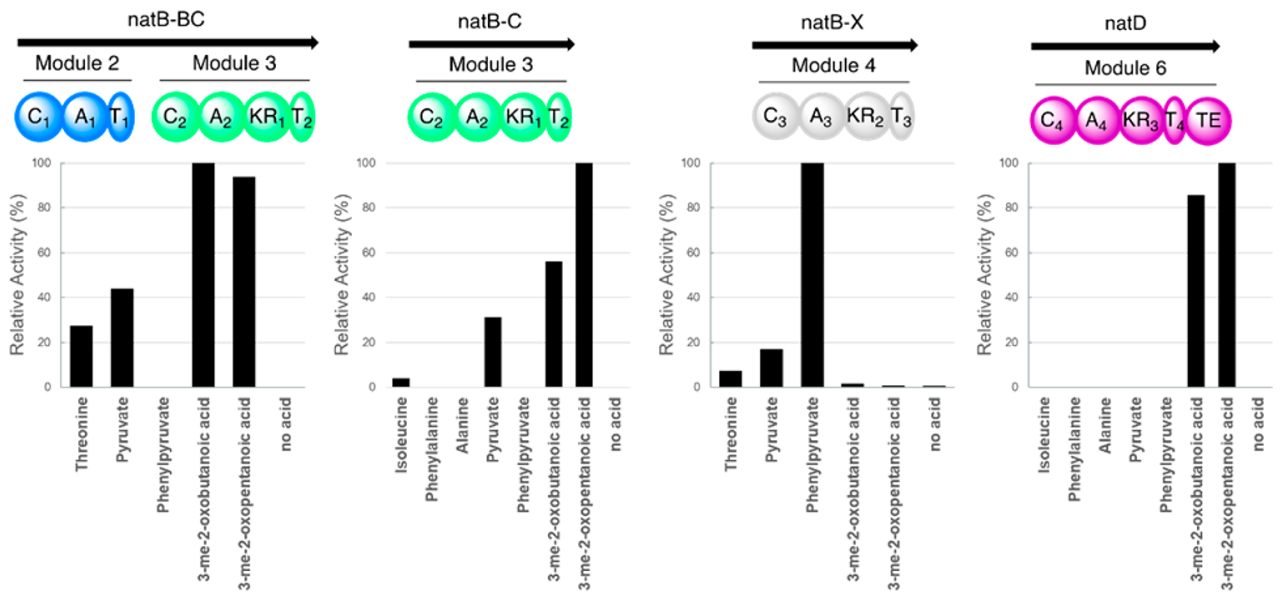

Figure 2. Analysis of NRPS adenylation domain activity. The four NRPS adenylation domains within the neoantimycin biosynthetic gene cluster were analyzed in vitro using ATP- $\left[{ }^{32} \mathrm{P}\right] \mathrm{PPi}$ exchange assays to verify utilization of the predicted substrate for each module. Values are displayed as relative activity normalized to 3-me-2-oxobutanoic acid (NatB-BC), 3-me-2-oxopentanoic acid (NatB-C), phenylpyruvate (NatB-X), and 3-me-2oxopentanoic acid ( $\mathrm{NatD})$ dependent exchange corresponding to approximately $250 \mathrm{k} \mathrm{cpm}$.

Here, we report the identification, cloning, and heterologous expression of the neoantimycin biosynthetic gene cluster from Streptomyces orinoci NRRL B-3379. We use our heterologous expression platform to establish the functionality of a transacting ketoreductase, NatF, and type II thioesterase, NatG, and combined with in vitro analyses, we also demonstrate that the geminal dimethyl moiety originates from an iteratively and cisacting methyltransferase domain within the NatC PKS. On the basis of our analyses, a biosynthetic pathway for neoantimycins is proposed and in the longer term our genetically tractable neoantimycin production platform can be used to facilitate the rapid generation of novel neoantimycin analogs.

\section{RESULTS AND DISCUSSION}

Identification of the Neoantimycin (nat) Biosynthetic Gene Cluster. The nat BGC was previously identified in the genome of S. orinoci NRRL B-3379; however, neither its DNA sequence nor the genome sequence was made publically available. ${ }^{11}$ The reported nat BGC and proposed biosynthetic pathway deviated considerably from what is known about the production of the related compound, antimycin. Two proteins essential for the biosynthesis of the starter unit 3formamidosalicylate were missing: an orthologue of AntG, a discrete peptidyl carrier protein (PCP), and an orthologue of AntI, a constituent of the multicomponent oxygenase that converts tryptophan to 3 -aminosalicylate prior to $\mathrm{N}$-formylation by AntO. ${ }^{1,16}$ In addition, in the reported nat BGC, there was a redundancy of ketoreductase (KR) domains encoded by both a standalone gene $(n a t F)$ and in the unimodular PKS gene $(n a t C)$ and a redundancy of thioesterase domains encoded by both a standalone gene (nat $G$ ) and in the NRPS gene (natE). The sixth module encoded by the NRPS genes (natD and $n a t E$ ) further showed abnormities that this module lacked a typical condensation domain and the protein itself was disconnected such that the adenylation domain encoding gene would be divergently transcribed from the opposite strand of DNA (Figure S1).

The above peculiarities prompted us to resequence $S$. orinoci NRRL B-3379. The final genome assembly, which is available under GenBank accession PHNC01000000, revealed a $7,502,208$ bp chromosome represented over 44 contigs. The nat BGC was identified by the genome mining package
antiSMASH $3.0,{ }^{17}$ whose identity was easily corroborated by manual identification of gene products orthologous to AntFGHIJKLNO from $S$. albus, 9,18,19 which biosynthesize the 3-formamidosalicylate moiety ${ }^{15,16,20}$ present in both antimycins and compounds $1-3$.

The $\sim 38 \mathrm{~kb}$ nat BGC identified in this study harbors 18 genes with the deduced functionalities shown in Table 1 and is organized into four apparent transcriptional units (Figure 1). Interestingly, we identified three previously unreported genes: natE, natQ and natI, which encode an $\mathrm{MbtH}$-like protein, a phosphopantetheinyl transferase, and the missing constituent of the multicomponent oxygenase involved in 3-formamidosalicylate biosynthesis, respectively (Table 1, Figure 1). In addition, we were unable to identify a KR domain within the deduced amino acid sequence for NatC and, strikingly, identified the gene encoding the NatD NRPS as a contiguous unbroken coding sequence that harbored the anticipated condensation domain (Figures 1 and $\mathrm{S} 1$ ). While it is formally possible that the differences between our nat BGC and that reported previously could result from genetic rearrangements and/or deletions, it is more likely to be the consequence of incomplete annotation and/or genome assembly error(s) considering the previous study demonstrated the strain to produce neoantimycins. $^{11}$

Verification of Adenylation Domain Substrate Specificity. To confirm that the various alkyl substitutions on the lactone ring are due to the promiscuity of the megasynthases, we tested the adenylation domain substrate specificity of the $\mathrm{NatB}$ and NatD NRPSs using an ATP/PPi exchange assay. $\mathrm{NatB}$ was truncated into three individual modules, NatB-B, -C, and $-\mathrm{X}$, that contain biosynthetic modules 2, 3, and 4, respectively, and were overproduced and purified from the $E$. coli strain BAP1. ${ }^{21}$ Purified NatB-C showed activation of 3methyl-2-oxobutanoic acid, 3-methyl-2-oxopentanoic acid, and pyruvate as expected by structural analysis of the naturally occurring neoantimycins, and NatB-X exhibited a strong activation of phenylpyruvate (Figure 2). Purified NatB-B curiously did not exhibit PPi exchange activity when incubated with its presumed substrate, L-threonine. However, the purified dimodule protein NatB-BC was able to activate L-threonine as well as 3-me-2-oxobutanoic acid, 3-me-2-oxopentanoic acid, and pyruvate (Figure 2). This suggests that individual 
truncation and purification of module 2 did not yield an active adenylation domain, albeit the protein seemed to be solubly expressed from E. coli (Figure S2). The terminal module harbored by NatD overproduced and purified as above and exhibited PPi activity when incubated with the tested substrates 3-methyl-2-oxobutanoic acid and 3-methyl-2-oxopentanoic acid. The demonstrated substrate specificities of four adenylation domains are all consistent with the known molecular structures of neoantimycins.

Cloning of the nat BGC and Heterologous Production of Neoantimycins by Streptomyces coelicolor. S. orinoci is genetically intractable, and therefore we pursued a heterologous expression strategy in order to study neoantimycin biosynthesis. We constructed a cosmid library and identified two overlapping cosmids, Cosmid 69 and Cosmid 813, which together span the entire nat BGC (Figure 1). In lieu of not capturing the entire gene cluster in one cosmid, we modified Cosmid 69 and Cosmid 813 to integrate into orthologous phage sites, which would abrogate the need to establish a contiguous gene cluster clone. Next, we replaced the native promoters of key loci in the gene cluster with strong constitutive ones to ensure expression of the gene cluster in a surrogate host (see Methods). In brief, Cosmid 69 was engineered such that natFG was expressed from the $\operatorname{rpsL}(\mathrm{XC})$ promoter (pRFSUL2), and Cosmid 813 was engineered such that natBCDE was expressed from the $\operatorname{rps} L(\mathrm{XC})$ promoter and natA was expressed from the ermE* promoter (pRFSUL3). On the basis of the promoter motifs recognized by its characterized ortholog $\left(\sigma^{\text {AntA }}\right)$, we predict that $\sigma^{\mathrm{NatA}}$ will activate expression of natQF $F^{\prime} G^{\prime} H I J L N O P^{22}$ Engineered cosmids pRFSUL2 and pRFSRUL3 were mobilized to $S$. coelicolor M1146, ${ }^{23}$ and chemical extracts prepared from cointegrant and parental strains were evaluated by LC-HRMS for the presence of neoantimycins. As we anticipated, molecular formulas for neoantimycins such as 1-3 were only observed in chemical extracts prepared from M1146 harboring both pRFSUL2 and pRFSUL3, but not in extracts generated from the empty M1146 strain (Figure S3). Taken together, these data unambiguously establish the identity of the genes required for the biosynthesis of neoantimycins.

Construction of a Chimeric Antimycin/Neoantimycin Biosynthetic Pathway in Streptomyces albus. Although we demonstrated effective neoantimycin production using our engineered cosmids and M1146, the strain does not sporulate well, grows relatively slowly, and harbors several antibiotic resistance markers, which limits the introduction of DNA in future experiments. We therefore selected S. albus J1074 as a heterologous production platform on the basis that it grows rapidly and has genome editing tools such as the CRISPR/Cas9 system readily available. ${ }^{24}$ Since $S$. albus J1074 is a native antimycin producer possessing the 3-formamidosalicylate biosynthetic machinery, we subcloned nat $A B C D E$ from Cosmid 813 into an E. coli-Streptomyces shuttle vector that additionally had natFG cloned under a constitutive ermE* promoter. The resulting plasmid containing natA-G was introduced into $S$. albus J1074 by conjugative transfer to generate the strain $4 \mathrm{c}-1$. Analysis of the culture extracts of $4 \mathrm{c}-1$ by LC-HRMS showed the heterologous production of all six of the neoantimycins that have previously been isolated from $S$. orinoci, and the titers of these compounds were comparable to those from the native producer (Figures 3 and S4). Successful combinatorial heterologous production of neoantimycins encouraged the creation of an unmarked heterologous host to facilitate future engineering efforts and remove background antimycin

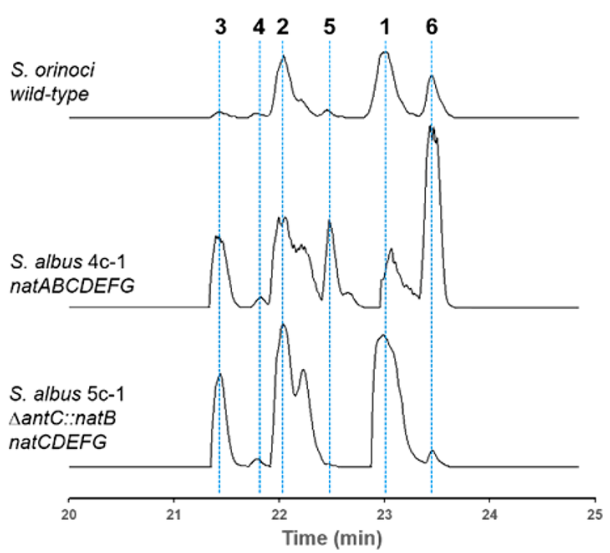

Figure 3. Heterologous production of neoantimycins. Extracted ion chromatograms show the production of neoantimycins by the wildtype $S$. orinoci and two engineered $S$. albus strains. The calculated mass with a $10 \mathrm{ppm}$ error tolerance was used. Calculated masses: $1, \mathrm{~m} / \mathrm{z}$ $699.3124[\mathrm{M}+\mathrm{H}]^{+} ; 2, m / z 685.2967[\mathrm{M}+\mathrm{H}]^{+} ; 3, \mathrm{~m} / z 671.2811[\mathrm{M}$ $+\mathrm{H}]^{+} ; 4, m / z 671.3180[\mathrm{M}+\mathrm{H}]^{+} ; 5, m / z 657.3018[\mathrm{M}+\mathrm{H}]^{+} ; 6, m / z$ $643.2861[\mathrm{M}+\mathrm{H}]^{+}$.

production. We used CRISPR/Cas9 genome editing to create an unmarked strain of S. albus J1074 (named 5c-1) in which ant $C$ was replaced by $n a t B$, and $e r m E^{*}$ p-driven natCDEFG was introduced into the intergenic space between antE and ant $F$ within the antimycin BGC (Figure 4). Chemical extracts prepared from $5 \mathrm{c}-1$ demonstrated its ability to produce neoantimycins with a titer similar to that of the native producer S. orinoci (Figure 3). These data demonstrate that the NatB NRPS efficiently interacts with 3-formamidosalycilate-S-AntG and is the first experimental evidence suggesting combinatorial bioengineering of ant-type depsipeptide biosynthesis may be possible.

Interestingly, the strain 5c-1 would promote the formation of a chimeric biosynthetic assembly line consisting of NatB and AntD (Figure 4), which would result in the production of 12membered ring ant-type depsipeptides related to JBIR-06. ${ }^{6} \mathrm{We}$ therefore closely inspected LC-HRMS data sets generated with the 5c-1 strain but were unable to detect molecular formulas consistent with variants related to JBIR-06, suggesting that both $\mathrm{NatB}$ and AntD do not interact to form a functional assembly line, or a dedicated thioesterase is needed to cyclize and release the 12-membered lactone ring. This conclusion is consistent with the lack of 12-membered ring compounds from the intermediate strains $5 \mathrm{c}-0$, in which ant $C$ was replaced by nat $B$ (Figure 4). In addition, the production of antimycins (ninemembered ring) by $5 \mathrm{c}-0$ and $5 \mathrm{c}-1$ was not detected, demonstrating the lack of interactions between $\mathrm{NatB}$ and AntD for antimycin synthesis through module skipping.

The Geminal-Dimethyl Moiety of Neoantimycins Is Generated by a cis-Acting Methyltransferase Domain That Functions Iteratively. The placement of the geminaldimethyl moiety observed at $\mathrm{C} 12$ of neoantimycins implicates involvement of the penultimate biosynthetic module encoded by the NatC PKS (Figure 1). Bioinformatics analysis of the NatC revealed the following domain composition, KS-AT-MTACP; however, the substrate specificity of AT could not be reliably predicted. ${ }^{25}$ Given the absence of other enzymes with predicted methyltransferase activity within the nat BGC, we hypothesized that the cis-acting MT domain generates the geminal-dimethyl moiety either by acting once upon a methylmalonate unit or twice upon a malonate unit. In order 


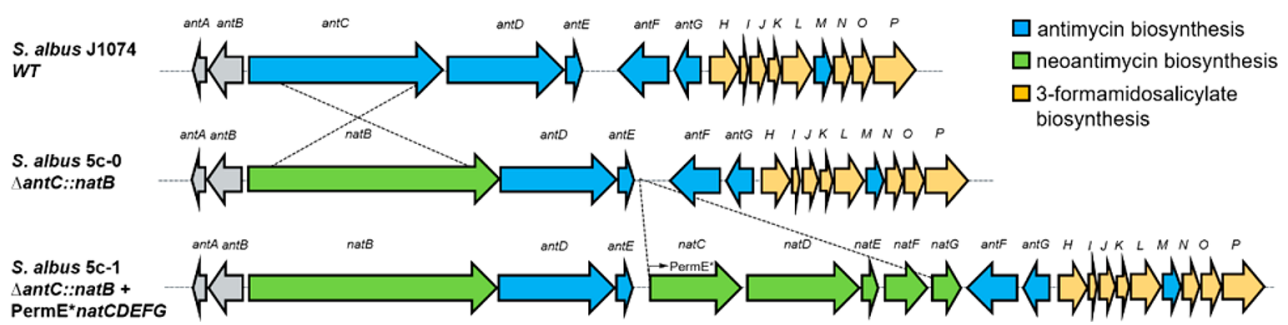

Figure 4. Construction of strain 5c-1 using CRISPR/Cas9. The gene antC in the antimycin BGC of $S$. albus J1074 is replaced by natB to generate an intermediate strain $5 \mathrm{c}-0$, followed by the insertion of natCDEFG to generate $5 \mathrm{c}-1$.

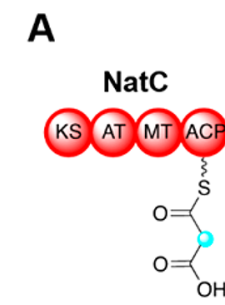

B

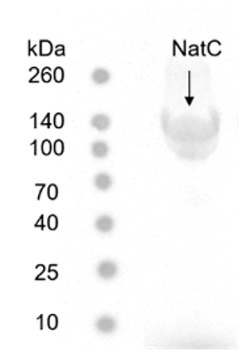

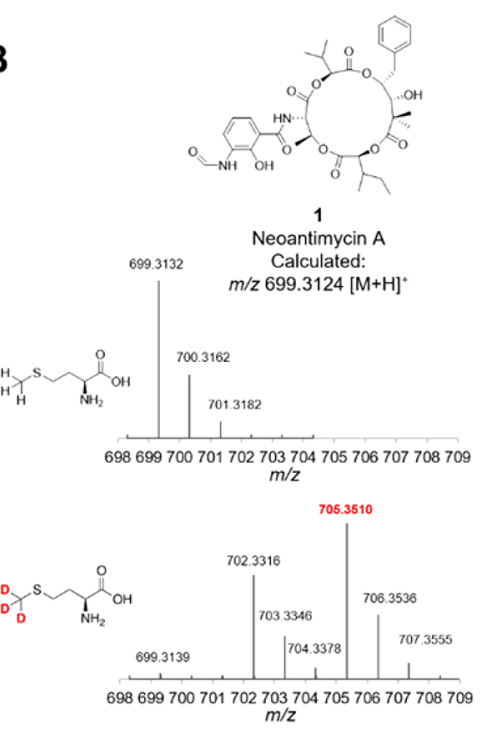

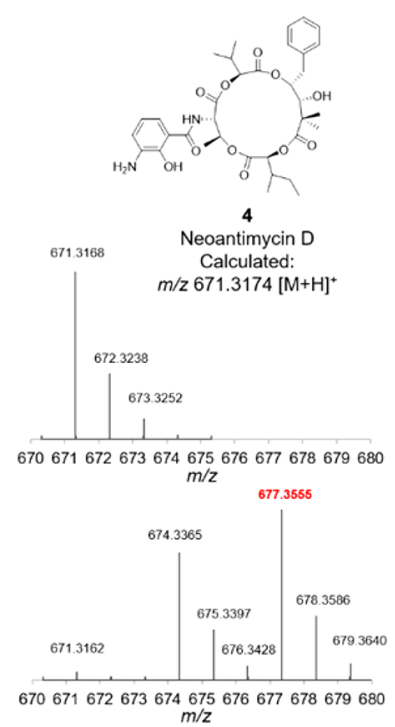

C
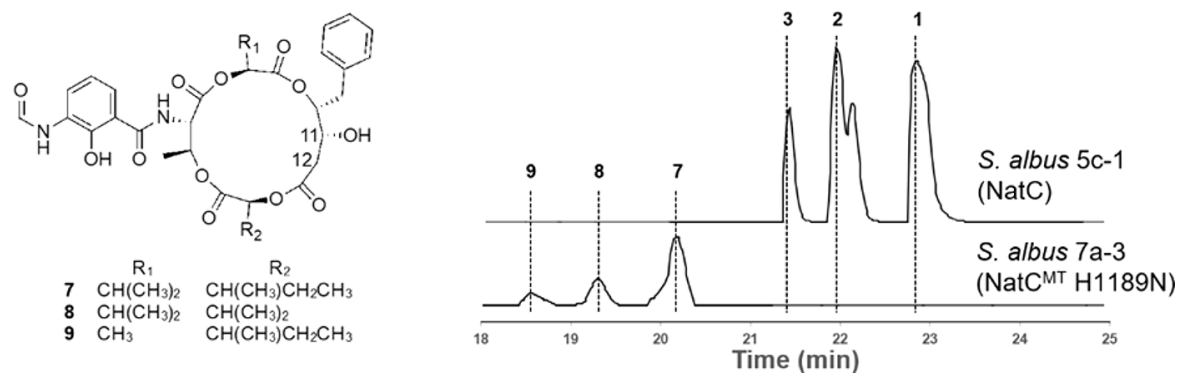

Figure 5. Characterization of NatC. (A) ${ }^{14} \mathrm{C}$ gel autoradiography assay showing the labeling of NatC by $\left[2-{ }^{14} \mathrm{C}\right]$ malonyl-CoA. (B) Selected isotopic peak patterns of neoantimycins produced by cultures fed with unlabeled L-methionine (top) and [methyl- $\left.\mathrm{d}_{3}\right]$ L-methionine (bottom). (C) Extracted ion chromatograms showing the production of neoantimycins and des-geminal dimethyl neoantimycins by engineered $S$. albus strains. The calculated mass with a $10 \mathrm{ppm}$ error tolerance was used. Calculated masses: $1, \mathrm{~m} / z 699.3124[\mathrm{M}+\mathrm{H}]^{+} ; 2, \mathrm{~m} / z 685.2967[\mathrm{M}+\mathrm{H}]^{+} ; 3, \mathrm{~m} / z 671.2811[\mathrm{M}+$ $\mathrm{H}]^{+} ; 7, m / z 671.2811[\mathrm{M}+\mathrm{H}]^{+} ; 8, m / z 657.2654[\mathrm{M}+\mathrm{H}]^{+} ; \mathbf{9}, \mathrm{m} / z 643.2498[\mathrm{M}+\mathrm{H}]^{+}$. The proposed structures of 7-9 are shown to the left.

to distinguish between these possibilities, we overexpressed and purified NatC using E. coli BAP1 and performed a ${ }^{14} \mathrm{C}$ gel autoradiography assay to determine if $\left[2-{ }^{14} \mathrm{C}\right]$ malonyl-CoA could be loaded onto the PKS. The results of this assay showed successful transfer of the radiolabel to $\mathrm{NatC}$, indicating that the NatC-AT domain was functional in vitro and capable of recognizing malonyl-CoA (Figure 5A). As this did not exclude the possibility that methylmalonyl-CoA could also be utilized, we then fed [methyl-d3] L-methionine (the precursor of the predicted methyl group donor, $S$-adenosylmethionine (SAM)) to $4 \mathrm{c}-1$. In this experiment, a single methylation would result in production of neoantimycins with molecular masses of $M+3$, whereas a dimethylation would produce molecular masses of $\mathrm{M}$ + 6. LC-UV analysis of the resultant chemical extracts showed that the fermentation product profiles remained the same upon the feeding of unlabeled and labeled L-methionine (Figure S5), and LC-HRMS analysis showed the presence of $M+6$ neoantimycins upon the feeding of [methyl-d3] L-methionine, indicating a dimethylation event. We also observed $M+3$ neoantimycins upon the feeding of labeled L-methionine, which is most likely a consequence of incorporation of one labeled and one unlabeled methyl group (Figure 5B).

To corroborate the above findings and to unambiguously determine the malonyl-CoA utilized by $\mathrm{NatC}$, we deactivated NatC-MT in 5c-1 by using CRISPR/Cas9 genome editing to replace a codon for a catalytically important histidine with asparagine $(\mathrm{H} 1189 \mathrm{~N}){ }^{26}$ LC-HRMS analysis of chemical extracts generated from the resulting mutant strain (named 7a-3) showed the absence of neoantimycins and the presence of compounds with molecular masses consistent with a loss of the 
geminal dimethyl moiety (Figures 5C and S6). Subsequent MS/MS analysis of these compounds in comparison to neoantimycins was indicative of des-geminal dimethyl neoantimycins (Figures S7 and S8). Taken together, these data indicate that NatC utilizes malonyl-CoA and NatC-MT acts iteratively to generate the geminal dimethyl moiety.

NatG Is a Proofreading Thioesterase and NatF Is a trans-Acting Ketoreductase. We next probed the "redundant" gene products NatG and NatF encoded in the nat BGC in comparison to the ant BGC. On the basis of our reannotation of nat $\mathrm{BGC}$, we propose that, similar to AntM, $\mathrm{NatF}$ is likely a trans-acting ketoreductase that is responsible for the apparent regiospecific ketoreduction at C11. Further bioinformatics analysis of NatG shows that it belongs to InterPro Family IPR01223, which indicates that NatG is likely a proofreading or type II thioesterase. Many PKS and NRPS biosynthetic gene clusters harbor a gene specifying a type II thioesterase, where they have been shown to increase production levels by removal of aberrant thioester intermediates from the assembly line. ${ }^{27} \mathrm{We}$ chose to establish the functionality of NatG and NatF in vivo and did so with our $S$. albus-based neoantimycin production platform.

Two cosmids harboring either natABCDEF or nat $A B C D E G$ were constructed and mobilized to $S$. albus J1074 to generate strains $4 c-2$ and $4 c-3$, respectively. Chemical extracts from the resulting strains were analyzed by LC-UV and compared to the extracts generated from S. albus J1074 harboring natABCDEFG. Neoantimycins were still produced in the absence of nat $G$ albeit at a reduced titer, which is consistent with our hypothesis that $\mathrm{NatG}$ is a proofreading thioesterase (Figure 6). Conversely,
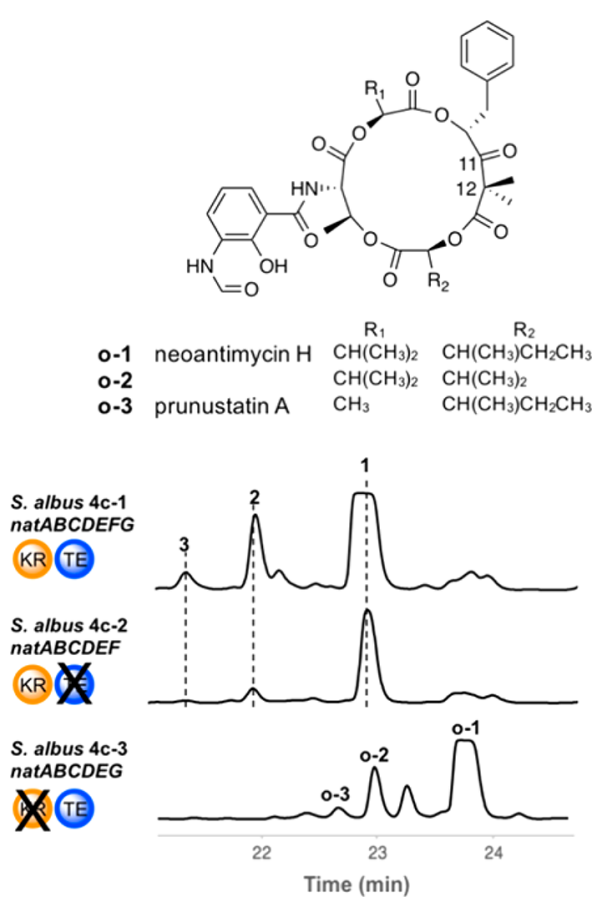

Figure 6. Characterization of NatF and NatG. HPLC-UV analysis of neoantimycins produced by engineered $S$. albus strains expressing different combinations of natF and nat $G$. Chromatograms $(320 \mathrm{~nm})$ show neoantimycin (1-3) production by $S$. albus $4 \mathrm{c}-1$ expressing nat $A B C D E F G$ and reduced titers of neoantimycins by $S$. albus $4 \mathrm{c}-2$ omitting nat $G$ that encodes a type II thioesterase. A new set of neoantimcyin derivatives was produced by $S$. albus $4 \mathrm{c}-3$ omitting nat $F$ that encodes a ketoreductase. neoantimycins were not observed in chemical extracts generated in the absence of natF; instead, a suite of compounds with UV absorption spectra identical to those of neoantimycins, but with shifted retention times, was observed (Figures 6 and S9). LC-HRMS and MS/MS analysis of these compounds were consistent with oxidized variants of neoantimycins, such as prunustatin $\mathrm{A}$ and neoantimycin $\mathrm{H}$ that were reported previously (Figures S10 and S11). ${ }^{13}$ The identity of $\mathbf{o - 3}$ was further confirmed by comparing to the authentic standard of prunustatin A. These results indicate that $\mathrm{NatF}$ is responsible for reduction of the keto group to a hydroxyl on C11. Since oxidized neoantimycins have shown interesting biological activities with prunustatin A established as a downregulator of the molecular chaperone $\mathrm{BiP} / \mathrm{GRP} 78,{ }^{12}$ this work has also generated a useful strain that produces C11 oxidized neoantimycins exclusively.

Proposed Biosynthetic Pathway for Neoantimycins. On the basis of the above in vivo and in vitro analysis, we propose a complete neoantimycin biosynthetic pathway as follows (Figure 1): it begins with the opening of the indole ring of tryptophan by a tryptophan 2,3-dioxyngenase (NatN) to produce $N$-formyl-L-kynurenine, which is converted to anthranilate by a housekeeping $\mathrm{N}$-formylase or $\mathrm{NatO}$ and a kynureninase (NatP). Anthranilate is then activated by an acylACP ligase, $\mathrm{NatF}^{\prime}$, and loaded into its cognate carrier protein, $\mathrm{NatG}^{\prime}$, followed by conversion to 3 -aminosalicylate by a multicomponent oxygenase NatHIJKL and $\mathrm{N}$-formylation by NatO. 3-Formamidosalicyl-S-NatG' serves as a starting unit for the hybrid NRPS/PKS machinery and is first presented to the NatB NRPS. NatB possesses three modules organized as follows: C1-A1-T1-C2-A2-KR1-T2-C3-A3-KR2-T3. The A1 domain activates and loads L-Thr onto T1 followed by condensation with 3-formamidosalicylate by $\mathrm{C} 1$ to form an amide bond. The A2 domain activates and loads pyruvate, 3methyl-2-oxobutanoic acid, or 3-methyl-2-oxopentanoic acid onto T2, which is subsequently stereospecifically reduced by KR1 and condensed with L-Thr by $\mathrm{C} 2$. The A3 domain activates and loads phenyl pyruvate onto $\mathrm{T} 3$, which is stereospecifically reduced by KR2 and condensed with the aminoacyl thioester attached to T2. The NatC PKS harbors one module organized as follows: KS-AT-MT-ACP. AT transfers malonate to ACP followed by installation of the geminal dimethyl group by MT. Next, KS catalyzes decarboxylative condensation between geminal dimethyl malonate and the aminoacyl thioester on T3 of NatB. The NatD NRPS harbors one module organized as follows: C4-A4-KR3-T4-TE. The A4 domain activates and loads 3-methyl-2-oxobutanoic acid or 3methyl-2-oxopentanoic acid onto $\mathrm{T} 4$, which is stereospecifically reduced by KR3 and condensed with the aminoacyl thioester attached to NatC-ACP prior to macrolactone cyclization and release of the 15-membered ring by NatD-TE. The trans-acting ketoreductase NatF reduces the $\mathrm{C} 11$ ketone to a hydroxyl, most likely acting on an intermediate tethered to the assembly line. In conclusion, our characterization and strain development within this work pave the way for rational reprogramming of the neoantimycin assembly line toward the biosynthesis of neoantimycin analogs.

\section{METHODS}

Growth Media, Strains, and Reagents. Escherichia coli strains were cultivated on Lennox agar (LA) or broth (LB), and Streptomyces strains were propagated on mannitol-soya flour agar or broth. ${ }^{28}$ Culture media were supplemented with antibiotics as required at the 
following concentrations: apramycin $(50 \mu \mathrm{g} / \mathrm{mL})$, carbenicillin (100 $\mu \mathrm{g} / \mathrm{mL})$, hygromycin $(75 \mu \mathrm{g} / \mathrm{mL})$, kanamycin $(50 \mu \mathrm{g} / \mathrm{mL})$, and nalidixic acid $(25 \mu \mathrm{g} / \mathrm{mL})$. Chemicals and media were purchased from Fisher Scientific or Sigma-Aldrich unless otherwise stated. Phusion High-Fedelity PCR Master Mix (NEB) was used for PCR reactions. Restriction and ligation enzymes were purchased from New England Biolabs unless otherwise stated. Oligonucleotides were purchased from Integrated DNA Technologies and are described in Table S1. The DNA constructs and bacterial strains used in this study are listed in Tables S2 and S3, respectively.

Genome Sequencing. S. orinoci NRRL B-3329 was obtained from the United States Department of Agriculture ARS stock center. $S$. orinoci chromosomal DNA was sequenced by the Earlham Institute (Norwich, UK) using the Pacific Biosciences and Illumina MiSeq platforms. The hierarchical genome assembly process (HGAP) was applied to two RSII SMRT cells worth of sequencing data, which generated 44 contigs comprised of $\sim 7.5 \mathrm{Mb}$ of DNA sequence. In order to correct errors originating from PacBio sequencing, 14,938,895 250-bp paired-end Illumina reads were mapped to these contigs using the Geneious R8.1.19 implementation of Bowtie2. The final genome assembly is available under DDBJ/EMBL/GenBank accession PHNC01000000 and consists of 44 contigs comprised of 7,502,208 bp. The raw PacBio and Illumina reads are available under Short Read Archive accessions SRR6318812 and SRR6318811, respectively.

Cosmid Library Construction and Screening. A Supercos1 cosmid library was constructed from S. orinoci NRRL B-3329 genomic DNA partially digested with Sau3AI and packaged into Gigapack III XL phage according to the manufacturer's instructions (Agilent Technologies). One thousand cosmid clones were screened for an insert spanning the neoantimycin BGC by PCR using primers RFS468 and RFS469 (which target the $3^{\prime}$ end of natB and $5^{\prime}$ end of natC, respectively) and RFS519 and RFS520 (which target natG; Table S1). The resulting cosmids, Cosmid 69 and Cosmid 813, were insert-end sequenced using primers RFS184 and RFS185 and mapped onto the $S$. orinoci genome using BLAST 2.2.29+. ${ }^{29}$

Construction of pUC19-PAprP, pUC19-PHygP, and pRFSUL1. The recombineering PCR templates PAprP and $\mathrm{PHygP}$ were constructed as follows: (1) RFS406 and RFS407 were used to amplify the apramycin resistance gene and oriT from $\mathrm{pIJ} 773^{30}$ and the hygromycin resistance gene and oriT from pIJ10700. ${ }^{31}$ (2) RFS658 and RFS659 were used to PCR amplify the rpsL(XC) promoter from pCRISPomyces. $^{24,32}$ (3) RFS667 and RFS668 were used to PCR amplify the ermE* promoter from pSET152-ermEp, ${ }^{33}$ and (4) RFS663 and RFS664 were used to linearize pUC19. The resulting PCR products were restricted with DpnI, gel purified, and assembled using the NEB HiFi DNA Assembly kit. The resulting plasmids, pUC19PAprP and pUC19-PHygP, contained FRT site-flanked apramycin and hygromycin resistance genes, respectively, and divergently firing $r p s L(\mathrm{XC})$ and ermE* promoters. The plasmid pRFSUL1 was generated by RecET recombineering using E. coli strain GB05-red. ${ }^{34}$ Briefly, oligonucleotides RFS448 and RFS449 were used to PCR amplify a $\sim 2.2 \mathrm{~kb}$ fragment from pMS82, which contained the $\Phi B T 1$ integrase, attP, and a hygromycin resistance gene. ${ }^{35}$ Recombineering with this PCR fragment replaced the neo/kan resistance gene present on the backbone of Supercos 1 to generate pRFSUL1. The $\Phi B T 1$ integrase, attP, and a hygromycin resistance gene is released from pRFSUL1 by a SspI restriction digest and can be used to recombineer Supercos1 clones of interest for integration into Streptomyces chromosomes. The DNA sequences for pUC19-PAprP, pUC19PHygP, and pRFSUL1 are available at http://www.ryanseipkelab. com/tools.html.

Cosmid Manipulations. Cosmid 69 and Cosmid 813 were engineered to integrate into $S$. coelicolor chromosomes using RecET recombineering with E. coli GB05-red and a $\sim 5.2 \mathrm{~kb}$ and $5.4 \mathrm{~kb} S \mathrm{spI}$ restriction fragment from pRFSUL1 and $\mathrm{pIJ} 10702,{ }^{36}$ respectively. The resulting cosmids were named Cosmid 69-ФBT1 and Cosmid 813ФC31. RecET recombineering with pUC19-PAprP and oligonucleotides DT133 and DT134 was used to modify Cosmid 69-ФBT1 such that it only harbored natFGQF' $G^{\prime} H I J K L N O P$ and that natFG expression was driven by the $\operatorname{rps} L(\mathrm{XC})$ promoter. The resulting cosmid was named pRFSUL2. RecET recombineering with pUC19$\mathrm{PHygP}$ and oligonucleotides DT132 and DT135 was used to engineer Cosmid 813-ФC31 such that nat $A$ and natB were expressed from the ermE* and $r p s L(\mathrm{XC})$ promoters, respectively, to result in Cosmid813ФC31-mod. The hygR gene of the PHygP cassette was removed from Cosmid $813-\Phi C 31$-mod by the Flp recombinase encoded by pCP20 as previously described ${ }^{30}$ to result in pRFSUL3.

Construction of Neoantimycin-Producing S. albus Strains. $S$. orinoci genomic DNA was used as a template for PCR amplification of $n a t F / G / F G$; the resulting PCR product was cloned into $\mathrm{PIB} 139^{37}$ by Gibson assembly. The resulting plasmid was then amplified by PCR and further cloned into the NsiI/CIP-digested cosmid 813 containing natA-E using Gibson assembly. Subsequently, the constructs were electroporated into E. coli WM6026 and used for conjugation with $S$. albus J1074. Transconjugants were selected by apramycin and kanamycin resistance and confirmed by PCR using the natE-Duet$\mathrm{F} /$ Duet-Mbth-R primers. The resulting strains were $4 \mathrm{c}-1,4 \mathrm{c}-2$, and $4 \mathrm{c}-$ 3. To construct the CRISPR/Cas9 generated strain 5c-1, the neoantimycin biosynthetic genes were introduced into the $S$. albus J1074 chromosome using the recently described pCRISPomyces-2 system. ${ }^{24}$ Two pCRISPomyces-2 plasmids (pJL129 and pJL134) were generated using Golden Gate and Gibson Assembly as previously described. $^{24}$ pJL129 was used to introduce natCDEFG under the control of the $\mathrm{ermE}^{*}$ promoter in between antE and antF. pJL134 was used to replace antC from the antimycin BGC with natB. First, pJL134 was mobilized to $S$. albus $\mathrm{J} 1074$ by cross-genera conjugation as previously described. ${ }^{28}$ Temperature sensitive pCRISPomyces-2 plasmid was removed from apramycin-resistant transconjugants by culturing at $37^{\circ} \mathrm{C}$. Replacement of antC by natB in the correct locus within the chromosome was verified by PCR and resulted in the generation of a strain we named 5c-0: S. albus J1074 $\Delta$ antC::natB. Next, pJL129 plasmid was mobilized to S. albus J1074 + PermE* natCDEFG and processed as above in order to generate a strain we named 5c-1: S. albus J1074 $\triangle$ antC::natB + PermE* natCDEFG. In order to introduce the H1189N mutation, a third pCRISPomyces -2 plasmid, pDS,90 was created by adapting pJL129 to insert natCDEFG with the mutant natC. To construct pDS90, pJL129 was digested with EcoR321 and BseJI restriction enzymes, and the resulting $26.7 \mathrm{~kb}$ linear plasmid was gel purified. A single base pair change in the MT domain was introduced by overlap PCR from $S$. orinoci genomic DNA using pJL129 EcoRV F/H1189N R and H1189N F/ pJL129_BseJ1_R. A three-piece Gibson assembly was used to introduce the overlapping PCR products to the digested pJL129 plasmid. The integrity of the resulting plasmid was verified by DNA sequencing and subsequently used as above to generate $S$. albus J1074 $\Delta$ antC::natB + PermE* natCDEFG + nat $C^{M T} \mathrm{H} 1189 \mathrm{~N}$, the methylation deficient neoantimycin producer, named 7a-3.

Analysis of S. coelicolor Strains. Engineered cosmids pRFSUL2 and PRFSUL3 were mobilized to S. coelicolor M1146 by cross-genera conjugation from E. coli ET12567/pUZ8002 as previously described. ${ }^{28}$ M1146 strains were cultured in $10 \mathrm{~mL}$ of LB while shaking $(200 \mathrm{rpm})$ at $30{ }^{\circ} \mathrm{C}$ for 3 days, at which point the entire culture was added to mannitol-soya flour broth $(50 \mathrm{~mL}$ in a 250 -mL flask) and incubated at $30{ }^{\circ} \mathrm{C}$ while shaking $(200 \mathrm{rpm})$. After 8 days of growth, bacterial cells were removed by centrifugation, and all of the culture supernatant was extracted once with two volumes of ethyl acetate and concentrated in vacuo. The residue was resuspended in $0.3 \mathrm{~mL}$ of methanol (100\%). Two microliters of methanolic extract were injected into a Bruker MaXis Impact TOF mass spectrometer equipped with a Dionex Ultimate 3000 HPLC exactly as previously described. ${ }^{33}$

Analysis of S. albus Strains. S. albus strains were first grown in a $2 \mathrm{~mL}$ of tryptic soy broth seed culture and inoculated at $1 \%$ inoculum into a $25 \mathrm{~mL}$ mannitol-soya flour broth. Cultures were grown for 5 days at $30{ }^{\circ} \mathrm{C}$ and $150 \mathrm{rpm}$. Mycelia were removed by centrifugation, and the supernatant was extracted with two volumes of ethyl acetate and dried with $\mathrm{MgSO}_{4}$ before rotary evaporation. Dried extracts were resuspended in methanol and analyzed via LC-HRMS or LC-UV-MS. LC-HRMS analysis was performed on an Agilent 6520 Accurate-Mass Q-TOF LC-MS, and LC-UV-MS analysis was performed on an Agilent 6120 Single Quadrupole LC/MS with a 1260 series DAD. Each 
instrument was equipped with an Agilent Eclipse Plus C18 column $(4.6 \times 100 \mathrm{~mm})$, and in each case a linear gradient of $25-95 \%$ $\mathrm{CH}_{3} \mathrm{CN}$ with $0.1 \%$ formic acid (v/v) over 20 min in $\mathrm{H}_{2} \mathrm{O}$ with $0.1 \%$ formic acid $(\mathrm{v} / \mathrm{v})$ at a flow rate of $0.5 \mathrm{~mL} / \mathrm{min}$ was used. A culture equivalent of $100 \mu \mathrm{L}$ was injected. A collision energy of $20 \mathrm{~V}$ was used for all HRMS/MS experiments.

Overproduction and Purification of Recombinant Protein. The NRPS components, NatB and NatD, and the PKS NatC were cloned and purified as follows. NatD and NatC were PCR amplified from $S$. orinoci genomic DNA as intact proteins. NatB was separated into three individual modules and PCR amplified as NatB-B, NatB-C, and NatB-X as well as a fourth construct containing the first two modules, NatB-BC from $S$. orinoci genomic DNA. Purified PCR constructs were cloned into either pET-30 or pET-24b using Gibson assembly or restriction enzyme digestion and quick ligation. Plasmids were verified by sequencing and transformed into BAP1 cells for protein production. Expression strains were grown in $0.7 \mathrm{~L}$ of $\mathrm{LB}$ supplemented with $50 \mu \mathrm{g} / \mathrm{mL}$ of kanamycin at $37^{\circ} \mathrm{C}$ and $250 \mathrm{rpm}$ until an $\mathrm{OD}_{600}$ of 0.5. Cultures were then put on ice for $10 \mathrm{~min}$ before induction with $120 \mathrm{mM}$ IPTG. Induction of gene expression lasted for $16 \mathrm{~h}$ at $16{ }^{\circ} \mathrm{C}$ and $200 \mathrm{rpm}$. The cells were then harvested by centrifugation $\left(6000 \mathrm{rpm}, 15 \mathrm{~min}, 4{ }^{\circ} \mathrm{C}\right)$, and supernatant was removed. The pellet was resuspended in $30 \mathrm{~mL}$ of lysis buffer $(25 \mathrm{mM}$ HEPES, pH 8, $0.5 \mathrm{M} \mathrm{NaCl}, 5 \mathrm{mM}$ imidazole) and homogenized using an Avestin homogenizer. The insoluble fraction was removed by centrifugation $\left(15,000 \mathrm{rpm}, 1 \mathrm{~h}, 4{ }^{\circ} \mathrm{C}\right)$, and the supernatant was filtered with a $0.45 \mu \mathrm{M}$ filter before batch binding. Ni-NTA resin (Qiagen) was added to the filtrate at $2 \mathrm{~mL} / \mathrm{L}$ of cell culture, and samples were allowed to nutate for $1 \mathrm{~h}$ at $4{ }^{\circ} \mathrm{C}$. The protein resin mixture was added to a gravity filter column, and the flow through was discarded. The column was then washed with approximately $24 \mathrm{~mL}$ of wash buffer ( $25 \mathrm{mM}$ HEPES, $300 \mathrm{mM} \mathrm{NaCl}, \mathrm{pH} 8)$ until untagged proteins were removed, determined by Bradford assay. Tagged protein was then eluted in approximately $18 \mathrm{~mL}$ of elution buffer $(25 \mathrm{mM}$ HEPES, $100 \mathrm{mM} \mathrm{NaCl}, 250 \mathrm{mM}$ imidazole, $\mathrm{pH}$ 8). Complete elution was determined by Bradford assay. Purified proteins were then concentrated and exchanged into appropriate buffer $(25 \mathrm{mM}$ HEPES, $100 \mathrm{mM} \mathrm{NaCl}, \mathrm{pH} 8)$ using Amicon ultra filter units. After two rounds of exchange and concentration, pure protein was removed, and glycerol was added to a final concentration of $8 \%$. Proteins were stored at $-80{ }^{\circ} \mathrm{C}$ or used immediately for in vitro assays.

Isotope-Labeled Precursor Feeding Experiments. S. albus J1074 4c-1 was cultured for the production of neoantimycins as described above, and $24 \mathrm{~h}$ after inoculation of the seed culture into 25 $\mathrm{mL}$ of MS with apramycin $(100 \mu \mathrm{g} / \mathrm{mL})$ and kanamycin $(100 \mu \mathrm{g} / \mathrm{mL})$, either unlabeled L-methionine or [methyl-d3] L-methionine was added to cultures to a final concentration of $1 \mathrm{~g} / \mathrm{L}$. Compound extraction and LC-HRMS analysis were performed as described above.

${ }^{14} \mathrm{C}$ Gel Autoradiography. Assays were performed in $10 \mu \mathrm{L}$ of 50 mM HEPES (pH 8.0) containing $1 \mathrm{mM}$ TCEP, $4 \mathrm{mM}$ ATP, $4 \mathrm{mM}$ $\mathrm{MgCl}_{2}, 1 \mathrm{mM}$ CoA, $0.13 \mathrm{mM}\left[2-{ }^{14} \mathrm{C}\right]$ malonic acid $(0.1 \mathrm{mCi} / \mathrm{mL}$; American Radiolabeled Chemicals), $25 \mu \mathrm{M}$ MatB (malonyl-CoA synthetase), and $90 \mu \mathrm{M}$ NatC. Reactions were incubated for $2 \mathrm{~h}$ at RT and quenched with an equal volume of $1 \times$ SDS sample buffer before SDS-PAGE analysis with a $4-15 \%$ TGX gel (Criterion). The gel was subsequently dried for $2.5 \mathrm{~h}$ at $50{ }^{\circ} \mathrm{C}$ and then exposed on a storage phosphor screen $(20 \times 25 \mathrm{~cm}$; Molecular Dynamics $)$ for 2-3 days. Phosphor images were captured using a Typhoon 9400 phosphorimager (Storage Phosphor mode, best resolution, $50 \mu \mathrm{m}$ resolution; Amersham Biosciences).

ATP-PPi Exchange Assays. Substrate specificity assays were performed in $100 \mu \mathrm{L}$ of reaction buffer $(50 \mathrm{mM}$ Tris- $\mathrm{HCl} \mathrm{pH} \mathrm{8,} 2 \mathrm{mM}$ $\mathrm{MgCl}_{2}$ ) containing $1 \mathrm{mM}$ TCEP, $5 \mathrm{mM} \mathrm{ATP,} 1 \mathrm{mM}$ tetrasodium pyrophosphate $\left(\mathrm{Na}_{4} \mathrm{PPi}\right), 5 \mathrm{mM}$ substrate, and $5 \mu \mathrm{M}$ enzyme. Before the addition of enzyme, $\mathrm{Na}_{4}\left[{ }^{32} \mathrm{P}\right]$-PPi was added to a final intensity of $\sim 2.5 \times 10^{6} \mathrm{cpm} / \mathrm{mL}$. Reactions were allowed to proceed for $2 \mathrm{~h}$ at 25 ${ }^{\circ} \mathrm{C}$ and then quenched by the addition of $500 \mu \mathrm{L}$ of charcoal (3.6\% w/ $\mathrm{v}$ activated charcoal, $\left.150 \mathrm{mM} \mathrm{Na} 4 \mathrm{PPi}, 5 \% \mathrm{HClO}_{4}\right)$. Samples were centrifuged, and supernatant was discarded. To remove residual free $\left.{ }^{[32} \mathrm{P}\right] \mathrm{PPi}$, the pellet was washed twice with wash solution $(0.1 \mathrm{M}$
$\mathrm{Na}_{4} \mathrm{PPi}, 5 \% \mathrm{HClO}_{4}$ ). The pellet was resuspended in $500 \mu \mathrm{L}$ of water and added to scintillation fluid at a final volume of $5 \mathrm{~mL}$. Radioactivity was measured using a Beckman LS 6500 scintillation counter.

\section{ASSOCIATED CONTENT}

\section{Supporting Information}

The Supporting Information is available free of charge on the ACS Publications website at DOI: 10.1021/acschembio.8b00298.

Oligonucleotides, DNA constructs, and bacterial strains used; annotation of the revised neoantimycin gene cluster; SDS-PAGE analysis of purified proteins; and compound characterization (PDF)

\section{AUTHOR INFORMATION}

\section{Corresponding Authors}

*E-mail: r.seipke@leeds.ac.uk.

*E-mail: wjzhang@berkeley.edu.

ORCID $\odot$

Wenjun Zhang: 0000-0001-9348-972X

\section{Author Contributions}

OThese authors contributed equally to this work.

Notes

The authors declare no competing financial interest.

\section{ACKNOWLEDGMENTS}

We thank K. Shin-ya (National Institute of Advanced Industrial Science and Technology, Japan) for providing prunustatin A standard for analysis. This work was financially supported by grants to W.Z. from the American Cancer Society (Grant RSG17-013-01-CDD), Alfred P. Sloan Foundation, the Chan Zuckerberg Biohub Investigator Program, and a grant from Biotechnology and Biological Sciences Research Council (BB/ N007980/1) to R.F.S. D.T. was supported by a University of Leeds 110 year anniversary studentship.

\section{REFERENCES}

(1) Liu, J., Zhu, X., Kim, S. J., and Zhang, W. (2016) Antimycin-type depsipeptides: discovery, biosynthesis, chemical synthesis, and bioactivities. Nat. Prod. Rep. 33, 1146.

(2) Joynt, R. and Seipke, R. F. (2018) A phylogenetic and evolutionary analysis of antimycin biosynthesis. Microbiology 164 28-39.

(3) Dunshee, B. R., Leben, C., Keitt, G. W., and Strong, F. M. (1949) The isolation and properties of antimycin A. J. Am. Chem. Soc. 71, 2436-2437.

(4) Tappel, A. L. (1960) Inhibition of electron transport by antimycin A, alkyl hydroxy naphthoquinones and metal coordination compounds. Biochem. Pharmacol. 3, 289-296.

(5) Tzung, S. P., Kim, K. M., Basañez, G., Giedt, C. D., Simon, J., Zimmerberg, J., Zhang, K. Y., and Hockenbery, D. M. (2001) Antimycin A mimics a cell-death-inducing Bcl-2 homology domain 3. Nat. Cell Biol. 3, 183-191.

(6) Ueda, J.-Y., Nagai, A., Izumikawa, M., Chijiwa, S., Takagi, M., and Shin-ya, K. (2008) A novel antimycin-like compound, JBIR-06, from Streptomyces sp. ML55. J. Antibiot. 61, 241-244.

(7) Cassinelli, G., Grein, A., Orezzi, P., Pennella, P., and Sanfilippo, A. (1967) New antibiotics produced by streptoverticillium orinoci, $n$. sp. Arch. Microbiol. 55, 358-368.

(8) Urushibata, I., Isogai, A., Matsumoto, S., and Suzuke, A. (1993) Respirantin, a novel insecticidal cyclodepsipeptide from Streptomyces. J. Antibiot. 46, 701-703.

(9) Seipke, R. F., Barke, J., Brearley, C., Hill, L., Yu, D. W., Goss, R. J. M., and Hutchings, M. I. (2011) A Single Streptomyces Symbiont 
Makes Multiple Antifungals to Support the Fungus Farming Ant Acromyrmex octospinosus. PLoS One 6, e22028-8.

(10) Vanner, S. A., Li, X., Zvanych, R., Torchia, J., Sang, J., Andrews, D. W., and Magarvey, N. A. (2013) Chemical and biosynthetic evolution of the antimycin-type depsipeptides. Mol. BioSyst. 9, 27122719.

(11) Li, X., Zvanych, R., Vanner, S. A., Wang, W., and Magarvey, N. A. (2013) Chemical variation from the neoantimycin depsipeptide assembly line. Bioorg. Med. Chem. Lett. 23, 5123-5127.

(12) Umeda, Y., Chijiwa, S., Furihata, K., Furihata, K., Sakuda, S., Nagasawa, H., Watanabe, H., and Shin-ya, K. (2005) Prunustatin A, a novel GRP78 molecular chaperone down-regulator isolated from Streptomyces violaceoniger. J. Antibiot. 58, 206-209.

(13) Salim, A. A., Cho, K.-J., Tan, L., Quezada, M., Lacey, E., Hancock, J. F., and Capon, R. J. (2014) Rare Streptomyces N-Formyl Amino-salicylamides Inhibit Oncogenic K-Ras. Org. Lett. 16, 50365039.

(14) Lampson, B. L., Pershing, N. L. K., Prinz, J. A., Lacsina, J. R., Marzluff, W. F., Nicchitta, C. V., MacAlpine, D. M., and Counter, C. M. (2013) Rare Codons Regulate KRas Oncogenesis. Curr. Biol. 23, $70-75$.

(15) Liu, J., Zhu, X., Seipke, R. F., and Zhang, W. (2015) Biosynthesis of antimycins with a reconstituted 3-formamidosalicylate pharmacophore in Escherichia coli. ACS Synth. Biol. 4, 559-565.

(16) Schoenian, I., Paetz, C., Dickschat, J. S., Aigle, B., Leblond, P., and Spiteller, D. (2012) An unprecedented 1,2-shift in the biosynthesis of the 3-aminosalicylate moiety of antimycins. ChemBioChem 13, 769773.

(17) Weber, T., Blin, K., Duddela, S., Krug, D., Kim, H. U., Bruccoleri, R., Lee, S. Y., Fischbach, M. A., Müller, R., Wohlleben, W., Breitling, R., Takano, E., and Medema, M. H. (2015) antiSMASH 3.0-a comprehensive resource for the genome mining of biosynthetic gene clusters. Nucleic Acids Res. 43, W237-W243.

(18) Seipke, R. F., Crossman, L., Drou, N., Heavens, D., Bibb, M. J., Caccamo, M., and Hutchings, M. I. (2011) Draft genome sequence of Streptomyces strain S4, a symbiont of the leaf-cutting ant Acromyrmex octospinosus. J. Bacteriol. 193, 4270-4271.

(19) Joynt, R., and Seipke, R. F. (2018) A phylogenetic and evolutionary analysis of antimycin biosynthesis. Microbiology 164, 2839.

(20) Sandy, M., Rui, Z., Gallagher, J., and Zhang, W. (2012) Enzymatic Synthesis of Dilactone Scaffold of Antimycins. ACS Chem. Biol. 7, 1956-1961.

(21) Pfeifer, B. A. (2001) Biosynthesis of Complex Polyketides in a Metabolically Engineered Strain of E. coli. Science 291, 1790-1792.

(22) Seipke, R. F., Patrick, E., and Hutchings, M. I. (2014) Regulation of antimycin biosynthesis by the orphan ECF RNA polymerase sigma factor $\sigma$ (AntA.). PeerJ 2, e253.

(23) Gomez-Escribano, J. P., and Bibb, M. J. (2011) Engineering Streptomyces coelicolor for heterologous expression of secondary metabolite gene clusters. Microb. Biotechnol. 4, 207-215.

(24) Cobb, R. E., Wang, Y., and Zhao, H. (2015) High-efficiency multiplex genome editing of Streptomyces species using an engineered CRISPR/Cas system. ACS Synth. Biol. 4, 723-728.

(25) Khayatt, B. I., Overmars, L., Siezen, R. J., and Francke, C. (2013) Classification of the Adenylation and Acyl-Transferase Activity of NRPS and PKS Systems Using Ensembles of Substrate Specific Hidden Markov Models. PLoS One 8, e62136-10.

(26) Skiba, M. A., Sikkema, A. P., Fiers, W. D., Gerwick, W. H., Sherman, D. H., Aldrich, C. C., and Smith, J. L. (2016) Domain Organization and Active Site Architecture of a Polyketide Synthase Cmethyltransferase. ACS Chem. Biol. 11, 3319-3327.

(27) Heathcote, M. L., Staunton, J., and Leadlay, P. F. (2001) Role of type II thioesterases: evidence for removal of short acyl chains produced by aberrant decarboxylation of chain extender units. Chem. Biol. 8, 207-220.

(28) Kieser, T. B., Buttner, M. J., Chater, M. J., and Hopwood, K. F. (2000) Practical Streptomyces Genetics, The John Innes Foundation, Norwich, UK.
(29) Camacho, C., Coulouris, G., Avagyan, V., Ma, N., Papadopoulos, J., Bealer, K., and Madden, T. L. (2009) BLAST+: architecture and applications. BMC Bioinf. 10, 421-9.

(30) Gust, B., Challis, G. L., Fowler, K., Kieser, T., and Chater, K. F. (2003) PCR-targeted Streptomyces gene replacement identifies a protein domain needed for biosynthesis of the sesquiterpene soil odor geosmin. Proc. Natl. Acad. Sci. U. S. A. 100, 1541-1546.

(31) Gust, B., Chandra, G., Jakimowicz, D., Yuqing, T., Bruton, C. J., and Chater, K. F. (2004) Lambda red-mediated genetic manipulation of antibiotic-producing Streptomyces. Adv. Appl. Microbiol. 54, 107128.

(32) Shao, Z., Rao, G., Li, C., Abil, Z., Luo, Y., and Zhao, H. (2013) Refactoring the Silent Spectinabilin Gene Cluster Using a Plug-andPlay Scaffold. ACS Synth. Biol. 2, 662-669.

(33) McLean, T. C., Hoskisson, P. A., and Seipke, R. F. (2016) Coordinate Regulation of Antimycin and Candicidin Biosynthesis. mSphere 1, e00305-16.

(34) Fu, J., Bian, X., Hu, S., Wang, H., Huang, F., Seibert, P. M., Plaza, A., Xia, L., Müller, R., Stewart, A. F., and Zhang, Y. (2012) Fulllength RecE enhances linear-linear homologous recombination and facilitates direct cloning for bioprospecting. Nat. Biotechnol. 30, 440446.

(35) Gregory, M. A., Till, R., and Smith, M. C. M. (2003) Integration site for Streptomyces phage phiBT1 and development of site-specific integrating vectors. J. Bacteriol. 185, 5320-5323.

(36) Yanai, K., Murakami, T., and Bibb, M. (2006) Amplification of the entire kanamycin biosynthetic gene cluster during empirical strain improvement of Streptomyces kanamyceticus. Proc. Natl. Acad. Sci. U. S. A. $103,9661-9666$.

(37) Wilkinson, C. J., Hughes-Thomas, Z. A., Martin, C. J., Böhm, I., Mironenko, T., Deacon, M., Wheatcroft, M., Wirtz, G., Staunton, J., and Leadlay, P. F. (2002) Increasing the efficiency of heterologous promoters in actinomycetes. J. Mol. Microbiol. Biotechnol. 4, 417-426. 